\title{
EXPERIMENTAL STUDY ON NOISE IDENTIFICATION OF MINIBUS REAR DRIVING AXLE WITH SOUND INTENSITY TECHNIQUE
}

\author{
Dequan Jin ${ }^{1,2, a}$, Wuwei Chen ${ }^{1, b}$, Huibin $\mathrm{Li}^{3, \mathrm{c}^{*}}$, Yue Zhang ${ }^{3, \mathrm{~d}}$ \\ ${ }^{1}$ School of Mechanical and Automotive Engineering, HEFEI UNIVERSITY OF TECHNOLOGY, \\ Hefei, Anhui, China, 230009 \\ ${ }^{2}$ Foton Daimler Automotive, Beijing,China, 101400 \\ ${ }^{3}$ School of Mechanical and Vehicular Engineering, Beijing Institute of Technology, Beijing, \\ China, 100081 \\ ajindequan@bfda.cn, ${ }^{b} \mathrm{cww} @ m a i l . h f . a h . c n,{ }^{c h}$ huibinli@163.com, ${ }^{\mathrm{d}} \mathrm{zh}$ hangyue_bit@163.com
}

\begin{abstract}
Keywords: rear driving axle; noise identification; sound intensity
Abstract. Firstly, based on the theories and methods of acoustic intensity measurement, the surface radiation noise of the rear driving axle of minibus was tested in the bench experiment. With the analysis of the experiment data, sound power was calculated to plot the noise distribution of the rear driving axle. It was found that the major noise source closed to the case of main reducer of the driving axle. The major noise frequencies are related to the gear meshing vibration. These results basically coincide with the acoustic array experimental results.
\end{abstract}

\section{Introduction}

The driving axle, which transmits the power and motion, is a critical assembly unit for automobile. When the driving axle works, it will produce noise and vibration which have a great influence on the NVH quality of the whole minibus. Domestic and foreign scholars have carried out a lot of experimental researches on the controlling noise and vibration of the driving axle, and their works include: (1)simple measurement of the noise and vibration;(2)technologies and techniques for controlling noise and vibration;(3) modal analysis ${ }^{[1-3]}$.

The frequencies of driving axle noise are discrete. In its spectrum, there one or several prominent peaks often appear. The literatures about the identification and control for the driving axle are relatively few. At present, the main methods and technologies for identifying the noises of automobiles, gearbox and engine are sound intensity measurement, acoustic holography measurement, acoustic arrays measurement, sound modal analysis, correlation analysis and partial coherence analysis, and so on ${ }^{[4-7]}$. In this paper, Firstly, based on the theories and methods of acoustic intensity measurement, the surface radiation noise of the rear driving axle is tested in the bench experiment. Then sound intensity contour map is used and sound power are calculated to plot the noise distribution of the driving axle and the main noise source is identified. These results will be compared with the sound array experimental results.

\section{Identification Theory of Acoustic Intensity}

The sound source with different natures produces particular sound signals, and the sound waves, in accordance with established laws of physics in space, propagate to form the sound field. The time history of sound pressure can be measured, at any point in the sound field, by using a non-directional characteristic microphone. This time history of sound represents the atmospheric pressure fluctuation in the position of the sensors, but this time history can not include much sound source information. Two microphones, arranged at the certain places in space with face to face or back to back manner, constitute a sound intensity apparatus, by which the sound pressure signal is measured, and the sound velocity would be obtained through processing the signal data. So the sound intensity could be calculated and sound source would also be identified. 
Shown as Fig.1, assume the space between two face-to-face acoustical sensors as $\Delta \mathrm{s}$. When the acoustical wave propagates along the $\mathrm{x}$ direction, there exists sound gradient between the two acoustical sensor's pressures.

Due to $\frac{\partial p}{\partial x}=-\rho_{0} \frac{\partial v}{\partial t}$, so sound velocity can be expressed as

$$
v(t)=-\frac{1}{\rho_{0}} \int \frac{\partial p(t)}{\partial x} d t
$$

When the space $\Delta \mathrm{s}$ is far less than sound wave $\lambda$, then $\frac{\partial p(t)}{\partial x}$ can be approximately expressed as $\frac{\left[p_{2}(t)-p_{1}(t)\right]}{\Delta s}$, and the equation (1) can be changed as

$$
v(t)=-\frac{1}{\rho_{0} \Delta s} \int\left[p_{2}(t)-p_{1}(t)\right] d t
$$

Furthermore, the sound pressure of the middle position of two acoustical sensors can be considered as the average of $p_{2}$ and $p_{1}$

$$
p(t)=\frac{p_{1}(t)+p_{2}(t)}{2}
$$

Then the instantaneous sound intensity along the $\mathrm{x}$ direction

$$
I_{x}(t)=p(t) v(t)=\frac{1}{2 \rho_{0} \Delta s}\left[p_{1}(t)+p_{2}(t)\right] \int\left[p_{2}(t)-p_{1}(t)\right] d t
$$

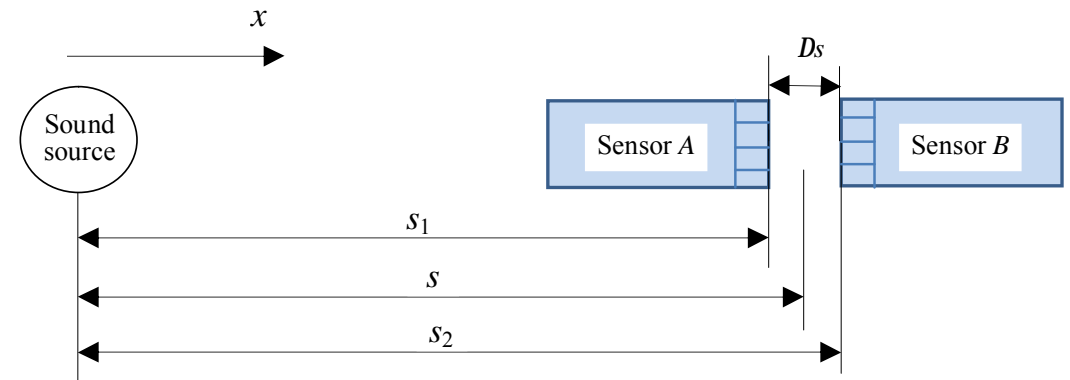

Fig.1 Position relation between sound source and two acoustical sensors

According the definition, the sound power on rectangular scanning surface can be expressed as

$$
W=\iint_{S} I_{n}(x, y) d x d y
$$

If the rectangular scanning surfaces are discretized, then the estimated values at discrete points can be expressed as

$$
W_{\text {Estimated }}=\sum_{i=1}^{n} \bar{I}_{n i} \Delta A_{i}
$$

Where $\bar{I}_{n i}$ is the time-averaged normal sound intensity at the $i$ th cell surface, $\Delta A_{i}$ is area of the $i$ th cell surface, and $N$ is total number of cell surfaces.

By using formula (6), the sound power of every scanning projection plane could be calculated, and furthermore the noise contribution from every components of driving axle could be identified.

\section{Bench Test for Identifying the Sound Source of Driving Axle}

Test system includes hardware and software, shown as Fig.2 and Fig.3. B\&K 3560C and PULSE Version8.0 were employed in the bench test. The microphone model is B\&K4197, its frequency response range of $20 \mathrm{~Hz}-40 \mathrm{kHz}$, and its sensitivity of $12.5 \mathrm{mv} / \mathrm{Pa}$ (at $250 \mathrm{~Hz}$ ). 

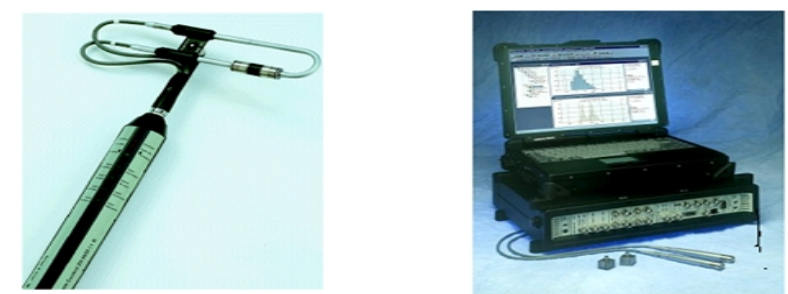

Fig.2 B\&K PULSE Version8.0 sound intensity measurement system

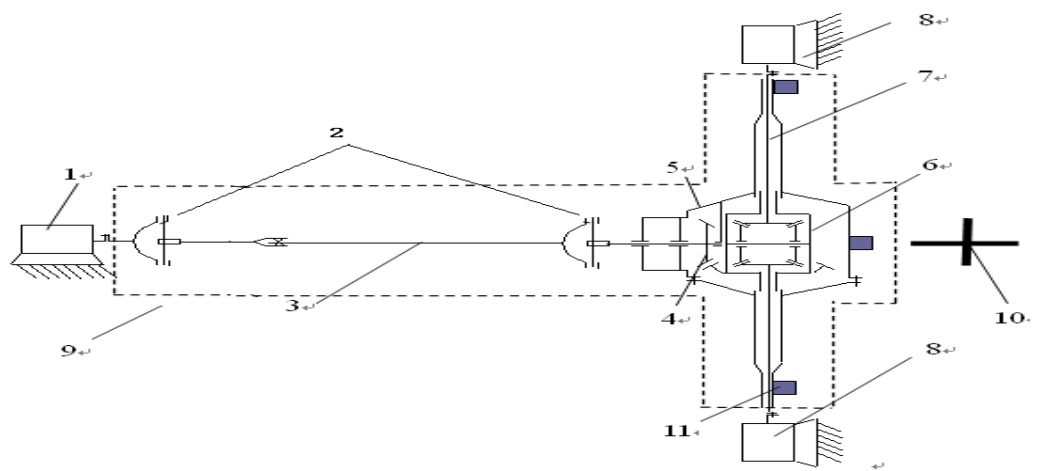

1- variable frequency driving motor; 2-univeral joint; 3-driving shaft; 4-main reducer; 5- driving axle case; 6- the differential; 7- differential axle; 8-loading motor; 9- scanning surface; 10-sound intensity measurement; 11-acceleration sensor

Fig. 3 Bench test layout for the noise identification of driving axle

Suppose 21 scanning envelope planes there when the acoustic intensity method was employed to identify the noise source of driving axle. The distance between the noise source surface and the supposed plane is $200 \mathrm{~mm}$, and the spacing distance between lines is $30 \mathrm{~mm}$. The number of total measurement points is 246 .

According to China National Standards, the driving axle is free of loads and the rotating speed of the driving shaft is $2304 \mathrm{r} / \mathrm{min}$, which corresponds to $50 \mathrm{~km} / \mathrm{h}$ of the minibus speed, for requirements of testing the NVH performance of minibus.

\section{Experiment Results}

Fig. 4 represents the noise signal measured at the position 20 centimeters apart from the surface of the driving axle. In this Figure, the noise concentrated at several frequencies such as $1367 \mathrm{~Hz}$, $1036.8 \mathrm{~Hz}$, which corresponding to four and three times of gear meshing frequency respectively, and so on.

By using the PULSE Version8.0 software, the noise signals from the acoustic intensity sensors were processed to obtain the sound intensity level distribution on the orthographical projection plane of the driving shaft-axle system, and also to obtain the sound intensity level distribution on the side projection plane of the driving shaft-axle, shown as from Fig.5 to Fig.9.

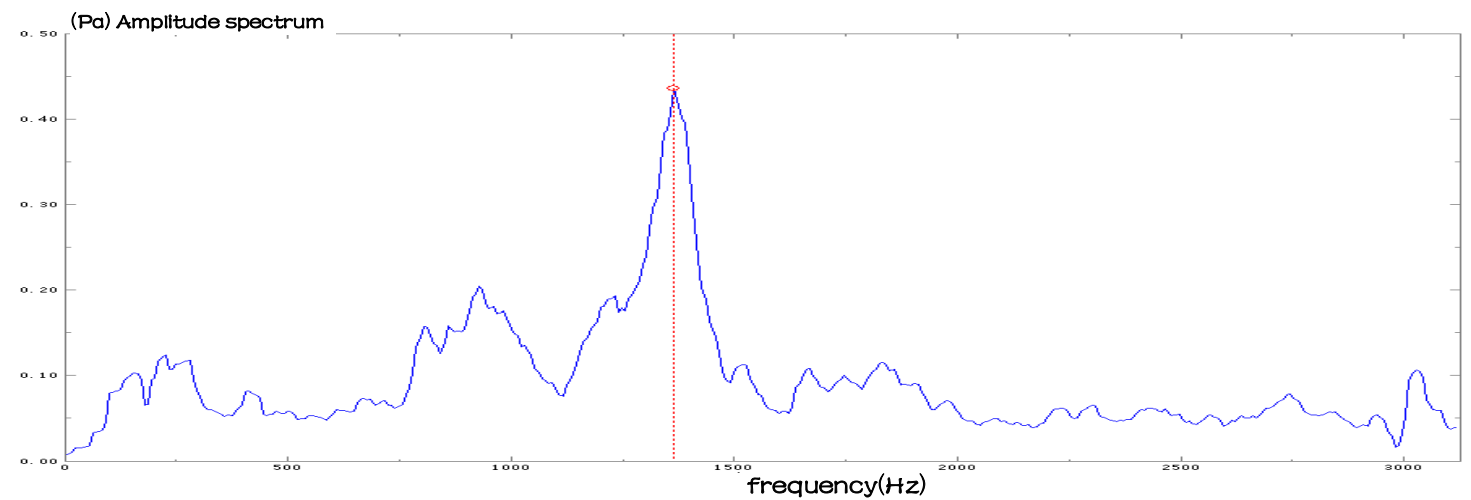

Fig.4 Far-field noise signals in frequency domain of driving axle 


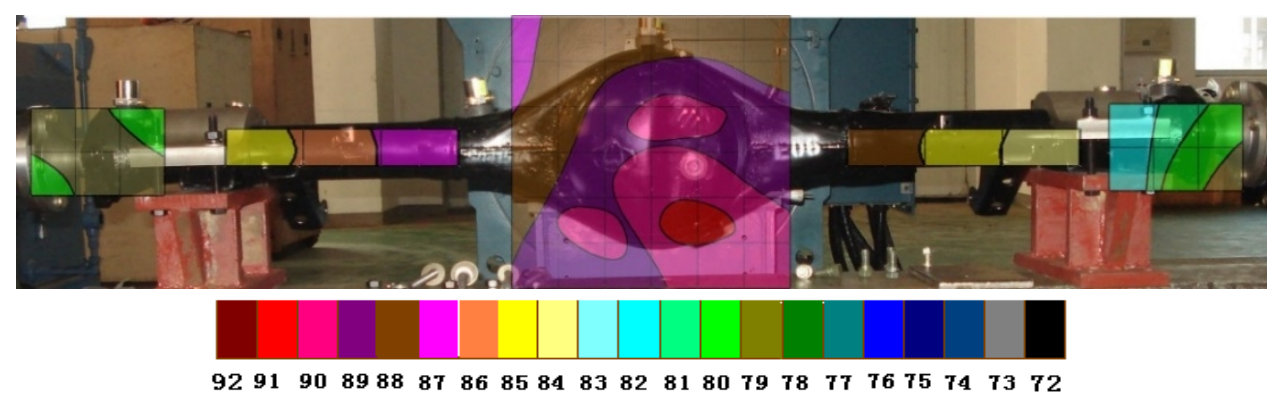

Fig. 5 Sound intensity cloud chart of rear driving axle's front orthographical projection plane

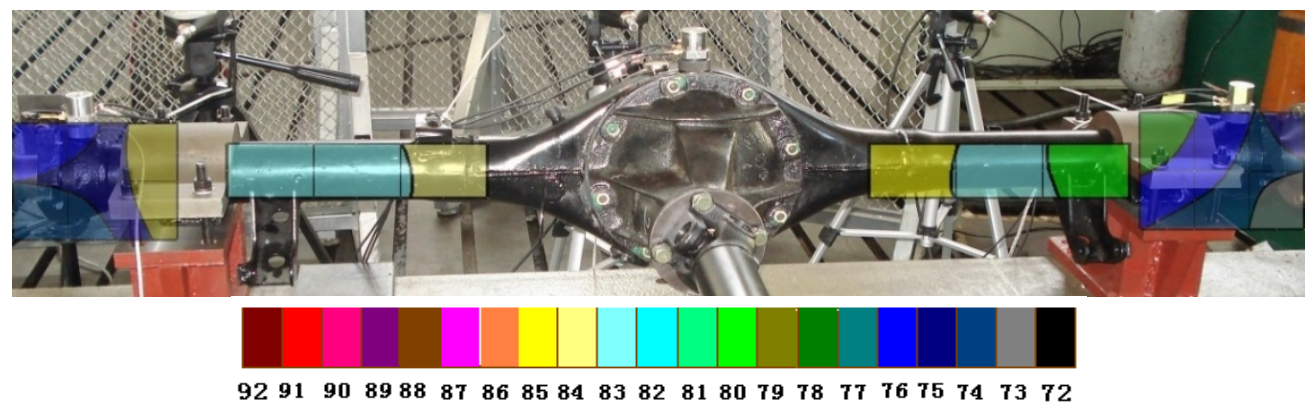

Fig.6 Sound intensity cloud chart of rear driving axle's rear orthographical projection plane

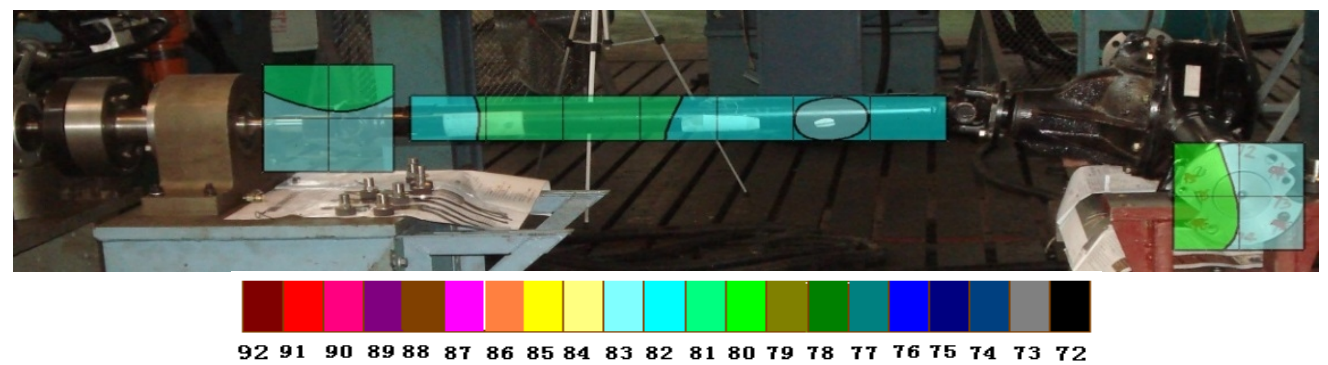

Fig.7 Sound intensity cloud chart of left side orthographical projection plane of transmitting shaft-rear driving axle's

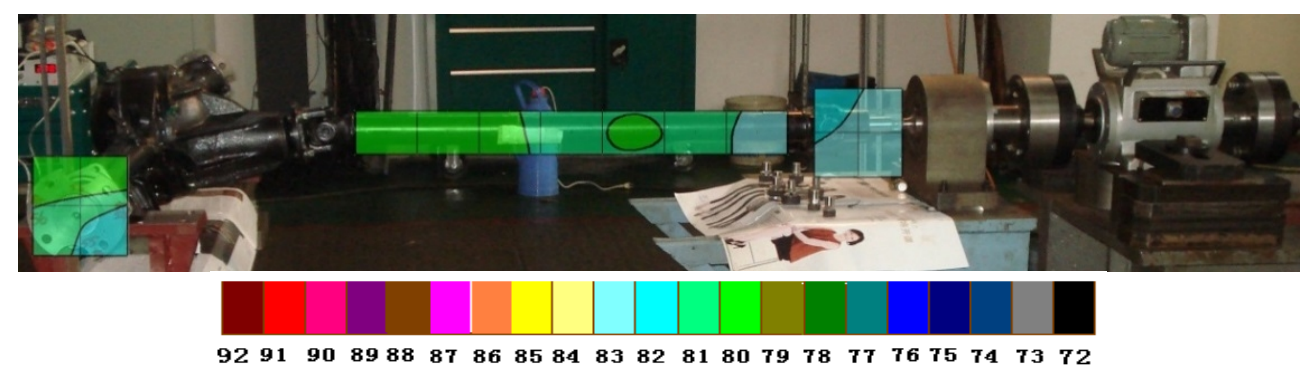

Fig. 8 Sound intensity cloud chart of right side orthographical projection plane of transmitting shaft-rear driving axle's

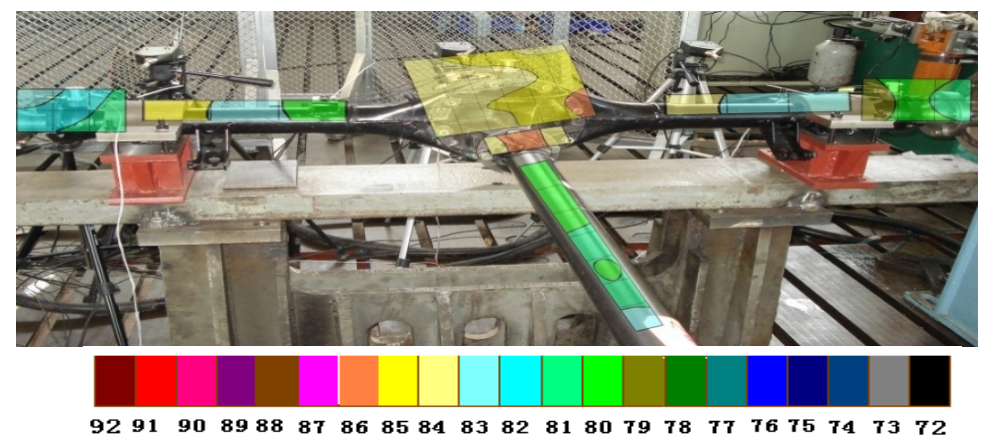

Fig.9 Sound intensity cloud chart of upper orthographical projection plane of transmitting shaft-rear driving axle's 
By using formula (6), the sound power level of every surface component is calculated according to the experimental data, and then the noise source is identified by the method of sound power ranking. The calculation results of sound power level for every orthographical projection plane are listed in Tab. 1. Fig.10 and Fig.11 are noise contribution distribution charts for all scanning planes. Fig. 12 is the total sound intensity level distribution on orthographical projection planes of driving axle, by using the acoustical array method.

Table 1 the noise contribution from different projection planes of transmitting shaft-rear driving axle

\begin{tabular}{|ccccc|}
\hline Main components & Projection planes & Sound power of planes $\left(10^{-5} \mathrm{~W}\right)$ & $\begin{array}{c}\text { Sound power } \\
\text { level/dB(A) }\end{array}$ & Percent \\
\hline Main reducer and & A,B,I & 73.53 & 88.66 & $73.24 \%$ \\
differential & 7.08 & 68.50 & $7.06 \%$ \\
Transmitting shaft & ZC1,ZC2,ZC3 & 4.76 & 66.78 & $4.74 \%$ \\
Differential axleI & ZA1,ZA2,ZA3 & 4.96 & 66.95 & $4.94 \%$ \\
Differential axleII & ZB1,ZB2,ZB3 & 2.50 & 63.98 & $2.49 \%$ \\
BearingI & C,E,K & 3.89 & 65.90 & $3.87 \%$ \\
BearingII & D,F,G & 3.68 & 65.66 & $3.66 \%$ \\
Bearing III & L,G,H & & & \\
\hline
\end{tabular}

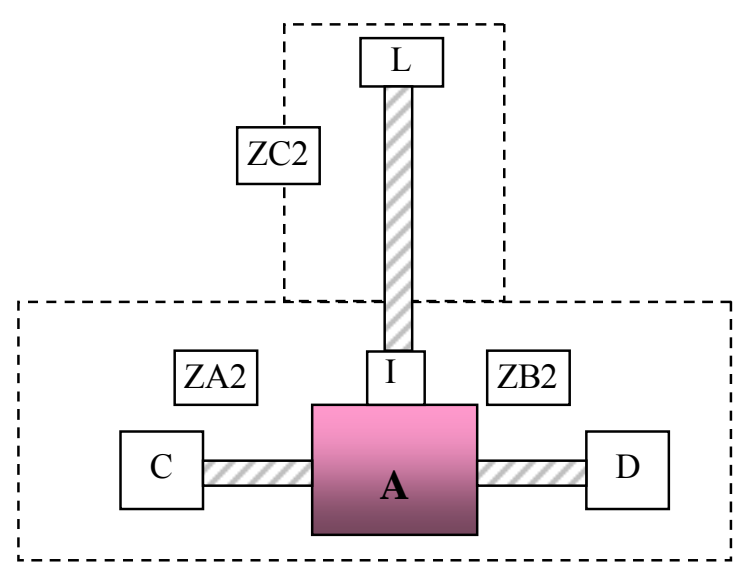

Fig.10 Noise contribution from overlooking projection plane (the Highest noise locate at plane A-main reducer)

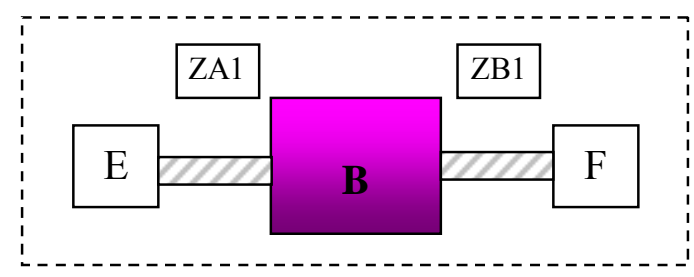

Fig.11 Noise contribution from face projection plane (the Highest noise locate at plane B-main reducer) 


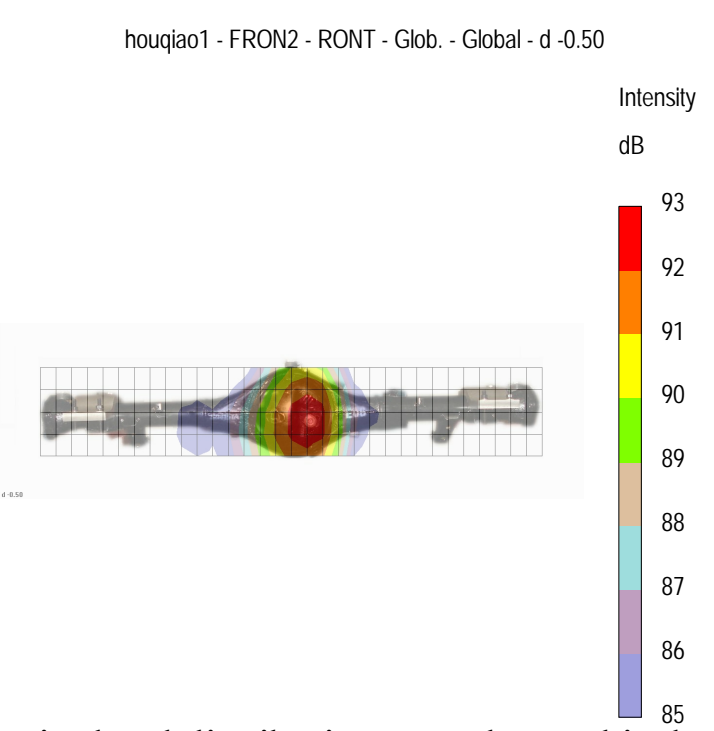

Fig. 12 Total sound intensity level distribution on orthographical projection plane of driving axle

It can be seen from Tab.1, Fig.11 and Fig.12, at the rotating speed of 2304rpm, the place with the maximum sound intensity level is located at the main reducer and differential, and its sound intensity level reaches $91.2 \mathrm{dBA}$. The noise contribution from the main reducer and differential is as many as $73.24 \%$ of the total sound power.

At the rotating speed of $2304 \mathrm{rpm}$, the identification result of the main sound resource, by using the acoustic array method, also indicated that the gear meshing from the main reducer is the main sound source.

\section{Conclusion}

The main reducer and differential, whose sound intensity level up to $91.2 \mathrm{dBA}$, are the main sound source of the driving axle system. The noise mechanism of the main reducer and differential must be studied and the noise must be reduced.

The noise identification by using the acoustic intensity method can clearly identify the main noise source, and the identification result coincides basically with the acoustic array method.

\section{Acknowledgements}

The authors express their gratitude to the Natural Science Foundation of China (50875022) for the financial support of the study.

\section{References}

[1] Huibin Li. Acoustic Array Identification Experiment and Analysis Report of the Driving axle of Minibus. Beijing: School of Mechanical and Vehicle Engineering, Beijing Institute of Technology, 2010.( in Chinese)

[2] Huibin Li, Mengxi Ning, Chengxia Zhang, Bo Yu. Experimental Study on the Noise Identification of the rear Driving Axle. Proceeding of the ISDEA,2012, pp.1255-1258.

[3] HuiHui Zhai. The noise and vibration analysis on the rear driving axle of a commercial vehicle [D]. Beijing Institute of Technology,2011. ( in Chinese)

[4] WeiKang JIANG, Qua WAN. Review and Outlook of Researches on Near-field Acoustical Holography Theories with Application. Journal of Mechanical Strength, 2005, 27(3), pp. 288-295. ( in Chinese)

[5] Guanglin Zhou. Scanning Sound Intensity Measurement Technique. Harbin Engineering University Publishing Press, 2007 ( in Chinese).

[6] Xiaoyu Jiang, Xiaoming Liang. Sound Intensity Technique and its Application in Automobile Engineering. Tsinghua University Publishing Press,2001 ( in Chinese). 
[7] Chengning ZHANG, Zaizhou WANG, Qiang SONG. Research of Noise Source Identification of Traction Motor System for Electric Vehicle Based on Microphone Array. Proceedings of the CSEE, 2008, 28(30), pp.109-112. ( in Chinese) 